\title{
Tidal Volume Fluctuations
}

National Cancer Institute

\section{Source}

National Cancer Institute. Tidal Volume Fluctuations. NCI Thesaurus. Code C63138.

Problem associated with the amount of gas that is inspired and expired during one respiratory cycle. 